\title{
QUANTUM MARKOV FIELDS
}

\author{
LUIGI ACCARDI \\ Centro Interdisciplinare Vito Volterra, II Università di Roma "Tor Vergata", \\ Via di Tor Vergata, 00133 Roma, Italy \\ accardi@volterra.uniroma2.it \\ FRANCESCO FIDALEO \\ Dipartimento di Matematica and Centro Interdisciplinare Vito Volterra, \\ II Università di Roma "Tor Vergata", \\ Via della Ricerca Scientifica, 00133 Roma, Italy \\ fidaleo@mat.uniroma2.it
}

Received 2 November 2001

\begin{abstract}
The Markov property for quantum lattice systems is investigated in terms of generalized conditional expectations. General properties of (particular cases of) quantum Markov fields, i.e. quantum Markov processes with multi-dimensional indices, are pointed out. In such a way, deep connections with the KMS boundary condition, as well as phenomena of phase transitions and symmetry breaking, naturally emerge.
\end{abstract}

Keywords: Noncommutative measure; integration and probability; quantum Markov processes; mathematical quantum statistical mechanics.

AMS Subject Classification: 46L53, 46L60, 60J99, 82B10

\section{Introduction}

Quantum Markov chains were introduced in Ref. 1 in the case of one-dimensional lattices. Since then, the problem to extend the theory to multi-dimensional lattices has remained open. Even if the intrinsic characterization of the Markov property in terms of the modular automorphisms group proposed in Ref. 5 is independent of the dimension, for a long time it did not bring, in the multi-dimensional case, to an easy controllable structure which would allow the construction of a multiplicity of nontrivial and physically meaningful examples as it happened in the one-dimensional case.

Let $\mathfrak{A}$ be a von Neumann algebra. Denote $F$ the family of finite parts of $\mathbb{Z}^{d}$, and let, two filtrations $\left\{\mathfrak{A}_{\Lambda}\right\},\left\{\mathfrak{A}_{\Lambda^{\prime}}\right\}$ of von Neumann subalgebras of $\mathfrak{A}$ be given for $\Lambda \in F$. Here $\Lambda^{\prime}$ denotes the set-theoretical complement of $\Lambda$, and both filtrations are increasing by inclusion. In order to avoid topological complications, it is convenient to think of $\mathfrak{A}$ as to the algebra of observables localized on a large but finite region 
$\hat{\Lambda}$ of the lattice. ${ }^{\mathrm{a}}$ In such a way, all the discussions are restricted to a finite volume and one could pass to the infinite volume limit in a second moment, see below.

Let $\varphi$ be a faithful normal state on $\mathfrak{A}$, and consider the canonical $\varphi$-conditional expectation firstly introduced in Ref. 3 ,

$$
E_{\mathfrak{A}_{\Lambda^{\prime}}}^{\varphi}: \mathfrak{A} \mapsto \mathfrak{A}_{\Lambda^{\prime}} .
$$

In the classical case we easily have

$$
\mathfrak{A}_{\Lambda^{\prime}}=\operatorname{Fix}\left(E_{\mathfrak{A}_{\Lambda^{\prime}}}^{\varphi}\right),
$$

where $\operatorname{Fix}\left(E_{\mathfrak{A}_{\Lambda^{\prime}}}^{\varphi}\right)$ denotes the set of all fixed points of $E_{\mathfrak{A}_{\Lambda^{\prime}}}^{\varphi}$.

In the quantum case, it was soon realized that the equality in (1.1) is too strong a condition. Then, in order to investigate the quantum Markov property for multi-dimensional models, we start with the following generalization.

First of all, to each $\Lambda \in F$ one associates another bounded set $\bar{\Lambda}$ with the property that $\Lambda \subset \bar{\Lambda}$.

Then, instead of (1.1), one requires the weaker condition

$$
\mathfrak{A}_{\bar{\Lambda}^{\prime}} \subset \operatorname{Fix}\left(E_{\mathfrak{A}_{\Lambda^{\prime}}}^{\varphi}\right)=: \mathfrak{A}_{\Lambda^{\prime}}^{\mathrm{fix}} \subset \mathfrak{A}_{\Lambda^{\prime}},
$$

where the last inclusion in (1.2) is usually proper.

By Ref. $3, \mathfrak{A}_{\Lambda^{\prime}}^{\text {fix }}$ is the largest subalgebra of $\mathfrak{A}_{\Lambda^{\prime}}$ with the property that the canonical $\varphi$-conditional expectation

$$
E_{\mathfrak{A}_{\Lambda^{\prime}}^{\mathrm{fix}}}^{\varphi}: \mathfrak{A} \mapsto \mathfrak{A}_{\Lambda^{\prime}}^{\mathrm{fix}}
$$

is a Umegaki conditional expectation onto $\mathfrak{A}_{\Lambda^{\prime}}^{\text {fix }}$. A constructive characterization of $E_{\mathfrak{A}_{\Lambda^{\prime}}}^{\varphi}$

$$
E_{\mathfrak{A}_{\Lambda^{\prime}}^{\mathrm{fix}}}^{\varphi}=\lim _{n} \frac{1}{n} \sum_{k=0}^{n-1}\left(E_{\mathfrak{A}_{\Lambda^{\prime}}}^{\varphi}\right)^{k}
$$

which always exists because of Ref. 17 . Suppose that the local algebras $\left\{\mathfrak{A}_{\Lambda}\right\}$ satisfy the additional conditions

$$
\mathfrak{A}_{\Lambda^{\prime}}^{\prime}=\mathfrak{A}_{\Lambda}, \quad \mathfrak{A}=\mathfrak{A}_{\Lambda} \vee \mathfrak{A}_{\Lambda^{\prime}} .
$$

Since condition (1.2) implies that, for each $a_{\bar{\Lambda}} \in \mathfrak{A}_{\bar{\Lambda}}, a_{\bar{\Lambda}^{\prime}} \in \mathfrak{A}_{\bar{\Lambda}^{\prime}}$, one has

$$
E_{\mathfrak{Q}_{\Lambda^{\prime}}}^{\varphi} a_{\bar{\Lambda}}\left(a_{\bar{\Lambda}} a_{\bar{\Lambda}^{\prime}}\right)=E_{\mathfrak{R}_{\Lambda^{\prime}}}^{\varphi} \operatorname{fix}_{\bar{\Lambda}}\left(a_{\bar{\Lambda}}\right) a_{\bar{\Lambda}^{\prime}}
$$

it follows that $E_{\mathfrak{A}^{\prime}}^{\varphi}$ fix $_{\Lambda^{\prime}}$ is uniquely determined by its restriction on $\mathfrak{A}_{\bar{\Lambda}}$.

Moreover, (1.3) implies that

$$
E_{\mathfrak{A}_{\Lambda^{\prime}}^{\text {fix }}}^{\varphi}\left(\mathfrak{A}_{\bar{\Lambda}}\right) \subset \mathfrak{A}_{\bar{\Lambda}} \wedge \mathfrak{A}_{\Lambda^{\prime}}=: \mathfrak{A}_{\partial \Lambda}
$$

a In such a situation, $\Lambda^{\prime}$ is understood as the complement in $\hat{\Lambda}$, that is $\Lambda^{\prime}=\hat{\Lambda} \backslash \Lambda$. 
which is an equivalent formulation of the classical Dobrushin-Markov property, see Refs. 2, 11-13. Conversely, given a $\varphi$-invariant Umegaki conditional expectation

$$
E_{\Lambda^{\prime}}: \mathfrak{A} \mapsto \mathfrak{B}_{\Lambda^{\prime}}
$$

satisfying the following property analogous to (1.2):

$$
\mathfrak{A}_{\bar{\Lambda}^{\prime}} \subset \mathfrak{B}_{\Lambda^{\prime}} \equiv \operatorname{Fix}\left(E_{\Lambda^{\prime}}\right) \subset \mathfrak{A}_{\Lambda^{\prime}},
$$

it follows by maximality that

$$
\mathfrak{B}_{\Lambda^{\prime}} \subset \mathfrak{A}_{\Lambda^{\prime}}^{\mathrm{fix}}
$$

If in addition (1.3) holds, then $E_{\Lambda^{\prime}}$ enjoys the Dobrushin-Markov property (1.4). Summing up, we get the following:

Theorem 1.1. Let $\varphi$ be a faithful state on $\mathfrak{A}$, and $\mathfrak{A}_{\Lambda}$ a von Neumann subalgebra of $\mathfrak{A}$ as above.

The following assertions are equivalent.

(i) The canonical $\varphi$-conditional expectation $E_{\mathfrak{A}_{\Lambda^{\prime}}}^{\varphi}$ satisfies the Markov property (1.2).

(ii) There exists a von Neumann subalgebra $\mathfrak{B}_{\Lambda^{\prime}}$ of $\mathfrak{A}$ such that

$$
\mathfrak{A}_{\bar{\Lambda}^{\prime}} \subset \mathfrak{B}_{\Lambda^{\prime}} \subset \mathfrak{A}_{\Lambda^{\prime}},
$$

and the canonical $\varphi$-conditional expectation $E_{\mathfrak{B}_{\Lambda^{\prime}}}^{\varphi}$ is a (surjective) Umegaki conditional expectation.

In the following we restrict ourselves to the case when $\mathfrak{B}_{\Lambda^{\prime}}$ coincides with $\mathfrak{A}_{\Lambda^{\prime}}^{\text {fix }}$, being the latter the largest subalgebra of $\mathfrak{A}_{\Lambda^{\prime}}$ invariant under the modular group $\sigma_{t}^{\varphi}$ of the state $\varphi$. We then have the monotonicity condition

$$
\Lambda_{1} \subset \Lambda_{2} \Rightarrow \mathfrak{A}_{\Lambda_{2}^{\prime}}^{\mathrm{fix}} \subset \mathfrak{A}_{\Lambda_{1}^{\prime}}^{\mathrm{fix}}
$$

which is equivalent to the standard projectivity condition

$$
\Lambda_{1} \subset \Lambda_{2} \Rightarrow E_{\mathfrak{A}_{\Lambda_{2}^{\prime}}^{\varphi}}^{\varphi}=E_{\mathfrak{A}_{\Lambda_{1}^{\prime}}^{\text {fix }}}^{\varphi}
$$

for the corresponding Umegaki conditional expectations.

Therefore, one can apply the formulation of Dobrushin's theory developed in Ref. 2, to the expected filtration $\left\{\mathfrak{A}_{\Lambda^{\prime}}^{\text {fix }}, E_{\mathfrak{A}_{\Lambda^{\prime}}^{\text {fix }}}^{\varphi}\right\}$.

If as in the case of quantum lattice systems, the local algebras are factorizable:

$$
\mathfrak{A}_{\Lambda_{1} \cup \Lambda_{2}} \cong \mathfrak{A}_{\Lambda_{1}} \bar{\otimes} \mathfrak{A}_{\Lambda_{2}}, \quad \Lambda_{1} \cap \Lambda_{2}=\emptyset,
$$

then

$$
\mathfrak{A}_{\partial \Lambda}=\mathfrak{A}_{\bar{\Lambda}} \wedge \mathfrak{A}_{\Lambda^{\prime}}
$$

and it is natural to define

$$
\partial \Lambda:=\bar{\Lambda} \cap \Lambda^{\prime} .
$$


Since $E_{\mathfrak{A}_{\Lambda^{\prime}}^{\text {fix }}}^{\varphi}$ is uniquely determined by its restriction $\mathcal{E}_{\Lambda^{\prime}}$ to $\mathfrak{A}_{\bar{\Lambda}}$, we can limit our considerations to the (finite volume) Umegaki conditional expectation

$$
\mathcal{E}_{\Lambda^{\prime}}: \mathfrak{A}_{\bar{\Lambda}} \mapsto \mathcal{R}\left(\mathcal{E}_{\Lambda^{\prime}}\right) \subset \mathfrak{A}_{\partial \Lambda}
$$

which was called transition expectation in Refs. 4 and 6 . If in addition the $\mathfrak{A}_{\bar{\Lambda}}$ have atomic centers (as in quantum lattice systems), then one can take advantage of the structure theory for such maps (see Refs. 14 and 15), and use it to extend to random fields the structure theorems proved for processes in Refs. 4-6. In particular, we associate to each (generalized) Markov field a potential which allows to recover uniquely the associated conditional expectations. We will show elsewhere that the conditions on this potential are explicit enough to allow the construction of a multiplicity of nontrivial examples.

\section{Notations}

To each site $i$ of the lattice $\mathbb{Z}^{d}$, we associate a fixed full matrix algebra $M_{i} \equiv \mathbb{M}_{k}(\mathbb{C})$, describing the observables localized in $i$. The algebra of all observables localized in a bounded region $\Lambda \subset \mathbb{Z}^{d}$ is then defined as

$$
\mathfrak{A}_{\Lambda}:=\otimes_{i \in \Lambda} M_{i} .
$$

Denote by $F$ the class of all finite subsets of $\mathbb{Z}^{d}$, and $\mathcal{F}$ the net generated by $F$ ordered by the inclusion between sets. We associate a fixed bounded "boundary" $\partial \Lambda \subset \Lambda^{\prime}$ to each bounded region $\Lambda$, where $\Lambda^{\prime}$ is the complement of $\Lambda$. In such a situation, we define

$$
\bar{\Lambda}:=\Lambda \cup \partial \Lambda
$$

For example,

$$
\partial \Lambda:=\left\{i \in \Lambda^{\prime}: \operatorname{dist}(i, \Lambda) \leq \delta\right\}
$$

for a fixed $\delta>0$ describing the range of the interaction.

We further write $\Lambda_{1} \subset \subset \Lambda_{2}$ if $\bar{\Lambda}_{1} \subset \Lambda_{2}$.

We consider the quasi-local algebra $\mathfrak{A}$ obtained by the $C^{*}$-inductive limit associated to the directed system $\left\{\mathfrak{A}_{\Lambda}\right\}_{\Lambda \in \mathcal{F}}$ with the natural embeddings

$$
\iota_{\Lambda, \hat{\Lambda}}: A_{\Lambda} \in \mathfrak{A}_{\Lambda} \rightarrow A_{\Lambda} \otimes I_{\hat{\Lambda} \backslash \Lambda} \in \mathfrak{A}_{\hat{\Lambda}}, \quad \Lambda \subset \hat{\Lambda} .
$$

In this situation we write

$$
\mathfrak{A}:=\otimes_{i \in \mathbb{Z}^{d}} M_{i},
$$

and extend (2.1) to arbitrary regions $\Lambda \subset \mathbb{Z}^{d}$. In these cases, the infinite tensor product is defined w.r.t. the unique $C^{*}$-cross norm. We often denote by $\iota_{\Lambda}: \mathfrak{A}_{\Lambda} \mapsto \mathfrak{A}$ the canonical injection of $\mathfrak{A}_{\Lambda}$ into $\mathfrak{A}$. The restrictions $\varphi_{\left\lceil\mathfrak{A}_{\Lambda}\right.}$ of a state $\varphi \in \mathcal{S}(\mathfrak{A})$ to local algebras $\mathfrak{A}_{\Lambda}$, will be denoted as $\varphi_{\Lambda}$. The reader is referred to Refs. 8, 9 and 28 for further details. 
By a (Umegaki) conditional expectation $E: \mathfrak{A} \mapsto \mathfrak{B} \subset \mathfrak{A}$ we mean a norm-one projection of the $C^{*}$-algebra $\mathfrak{A}$ onto a $C^{*}$-subalgebra (with the same identity $I) \mathfrak{B}$. The map $E$ is automatically a completely positive identity-preserving $\mathfrak{B}$-bimodule map, see Sec. 9 of Ref. 23. When $\mathfrak{A}$ is a matrix algebra, the structure of a conditional expectation is well-known, see Proposition 2.2 of Ref. 15 and Proposition 5 of Ref. 14 for the generalization to the case when $\mathfrak{A}$ and $\mathfrak{B}$ have atomic (possibly infinite-dimensional) centers.

Assume that $\mathfrak{A}$ is a matrix algebra and consider the (finite) sets $\left\{P_{i}\right\}$ and $\left\{Q_{j}\right\}$ of minimal central projections of $\mathfrak{A}$ and $\mathfrak{B}$ respectively. Denote $\mathfrak{A}_{i}:=\mathfrak{A}_{P_{i}}, \mathfrak{B}_{j}:=\mathfrak{B}_{Q_{j}}$, $\mathfrak{A}_{i j}:=\mathfrak{A}_{P_{i} Q_{j}}, \mathfrak{B}_{i j}:=\mathfrak{B}_{P_{i} Q_{j}}$, the corresponding von Neumann algebras. ${ }^{\mathrm{b}}$ Define:

(i) $\sigma_{i j}: \mathfrak{B}_{i j} \mapsto \mathfrak{B}_{j}$ the isomorphism reciprocal to the induction isomorphism $\mathfrak{B}_{j} \mapsto \mathfrak{B}_{i j}$

(ii) the column-Markovian matrix $\gamma:=\left[\gamma_{i j}\right]$ determined by

$$
E\left(P_{i}\right)=\sum_{j} \gamma_{i j} Q_{j}
$$

(iii) the conditional expectation $E_{i j}: \mathfrak{A}_{i j} \mapsto \mathfrak{B}_{i j}$ which is the zero-map whenever $\gamma_{i j}=0$, and

$$
E_{i j}(A):=\gamma_{i j}^{-1} P_{i} E(A)
$$

otherwise.

Then we obtain for the given conditional expectation,

$$
E(A)=\sum_{i, j} \gamma_{i j} \sigma_{i j}\left(E_{i j}\left(P_{i} Q_{j} A Q_{j}\right)\right) .
$$

Further, as $\mathfrak{A}_{i j}$ and $\mathfrak{B}_{i j}$ are finite dimensional factors, we have an explicit description of the conditional expectations $\left\{E_{i j}\right\}$, see Lemma 3.1 of Ref. 6 . Indeed, we easily decompose

$$
\mathfrak{A}_{i j}=N_{i j} \otimes \bar{N}_{i j},
$$

where $N_{i j} \cong \mathfrak{B}_{i j}$ and $\bar{N}_{i j} \cong \mathfrak{B}_{i j}^{\prime} \wedge \mathfrak{A}_{i j}$.

Then there exist states $\phi_{i j}$ on $\bar{N}_{i j}$ such that

$$
E_{i j}(a \otimes \bar{a})=\phi_{i j}(\bar{a}) a \otimes I .
$$

The case we are interested in, is precisely that corresponding to $\{i\}$ being a singleton. We have reported here the above general case for possible applications to more general situations.

\footnotetext{
${ }^{\mathrm{b}}$ Let $M$ be a von Neumann algebra acting on the Hilbert space $\mathcal{H}$, and $p \in M$ a self-adjoint projection. The reduced von Neumann algebra $M_{p}$ is defined as the von Neumann algebra $p M p$ acting on the Hilbert space $p \mathcal{H}$. Analogously, if $p \in M^{\prime}$, then the induced von Neumann algebra $M_{p}$ is the von Neumann algebra $p M$ acting on the Hilbert space $p \mathcal{H}$.
} 
Let $N \subset M$ be an inclusion of von Neumann algebras equipped with a normal faithful state on $M$. By the $\varphi$-conditional expectation we mean the identity-preserving normal faithful completely positive map

$$
E^{\varphi}: M \mapsto N
$$

defined in Ref. 3. Such a map preserves the state $\varphi$ and seems to be the natural generalization of the concept of conditional expectation in the sense of Umegaki, even if the former is not in general a norm-one projection.

Such a $\varphi$-conditional expectation is written as

$$
\pi_{\varphi_{\lceil N}}\left(E^{\varphi}(a)\right)=J_{\varphi_{\Gamma N}} P_{\varphi_{\lceil N}} J_{\varphi} \pi_{\varphi}(a) J_{\varphi} J_{\varphi_{\Gamma N}},
$$

where the $J$ are the Tomita antiunitary conjugations, and $P_{\varphi_{\Gamma N}} \in \pi_{\varphi}(N)^{\prime}$ is the cyclic projection relative to $\pi_{\varphi}(N)$, see e.g. Ref. 16 .

\section{The Quantum Markov Property on the Spin Algebra: An Intrinsic Definition}

Following the suggestion of Theorem 5.1 of Ref. 4, we show a characteristic of the Markov property in terms of the properties of canonical $\varphi$-conditional expectations, ${ }^{3}$ here denoted also as generalized conditional expectations, which are in our situation, canonical objects intrinsically associated to the local structure of the quasi-local algebra $\mathfrak{A}$, and the state $\varphi$ under consideration.

Definition 3.1. A locally faithful state $\varphi \in \mathcal{S}(\mathfrak{A})$ is said to be a Markov state if, for every pair of regions $\Lambda, \hat{\Lambda} \in F$ with $\Lambda \subset \subset \hat{\Lambda}$, we have

$$
E_{\hat{\Lambda} \backslash \Lambda}^{\varphi_{\hat{\Lambda}}}\left\lceil\mathfrak{A}_{\hat{\Lambda} \backslash \bar{\Lambda}}=\operatorname{id}_{\mathfrak{A}_{\hat{\Lambda} \backslash \bar{\Lambda}}} .\right.
$$

where $\varphi_{\hat{\Lambda}}$ is the restriction of $\varphi$ to $\mathfrak{A}_{\hat{\Lambda}}$, and $E_{\hat{\Lambda} \backslash \Lambda}^{\varphi_{\hat{\Lambda}}}: \mathfrak{A}_{\hat{\Lambda}} \mapsto \mathfrak{A}_{\hat{\Lambda} \backslash \Lambda}$ is the $\varphi_{\hat{\Lambda}}$-conditional expectation relative to the inclusion $\mathfrak{A}_{\hat{\Lambda} \backslash \Lambda} \subset \mathfrak{A}_{\hat{\Lambda}}$.

The ergodic limit

$$
E_{\hat{\Lambda} \backslash \Lambda}:=\lim _{n} \frac{1}{n} \sum_{k=0}^{n-1}\left(E_{\hat{\Lambda} \backslash \Lambda}^{\varphi_{\hat{\Lambda}}}\right)^{k}
$$

exists (see e.g. Refs. 17 and 19 for the most general situation) and is the conditional expectation onto the largest subalgebra of $\mathfrak{A}_{\hat{\Lambda} \backslash \Lambda}$ left invariant by the modular group $\sigma_{t}^{\varphi_{\hat{\Lambda}}}$. Furthermore by (3.1), $\mathfrak{A}_{\hat{\Lambda} \backslash \bar{\Lambda}} \subset \mathcal{R}\left(E_{\hat{\Lambda} \backslash \Lambda}\right)$, being the last range of $E_{\hat{\Lambda} \backslash \Lambda}$.

Lemma 3.2. With the above notations,

$$
E_{\hat{\Lambda} \backslash \Lambda}=\mathcal{E}_{\hat{\Lambda} \backslash \Lambda} \otimes \operatorname{id}_{\mathfrak{A}_{\hat{\Lambda} \backslash \bar{\Lambda}}},
$$

where

$$
\mathcal{E}_{\hat{\Lambda} \backslash \Lambda}:=E_{\hat{\Lambda} \backslash \Lambda}\left\lceil\mathfrak{A}_{\bar{\Lambda}}\right.
$$

is a conditional expectation of $\mathfrak{A}_{\bar{\Lambda}}$ onto a subalgebra of $\mathfrak{A}_{\partial \Lambda}$. 
Proof. It is enough to verify that $E_{\hat{\Lambda} \backslash \Lambda}\left(\mathfrak{A}_{\bar{\Lambda}}\right) \subset \mathfrak{A}_{\hat{\Lambda} \backslash \bar{\Lambda}}^{\prime} \wedge \mathfrak{A}_{\hat{\Lambda} \backslash \Lambda}$. Let $a_{\bar{\Lambda}} \in \mathfrak{A}_{\bar{\Lambda}}$ and $a_{\hat{\Lambda} \backslash \bar{\Lambda}} \in \mathfrak{A}_{\hat{\Lambda} \backslash \bar{\Lambda}}$. We get

$$
\begin{aligned}
E_{\hat{\Lambda} \backslash \Lambda}\left(a_{\bar{\Lambda}}\right) a_{\hat{\Lambda} \backslash \bar{\Lambda}} & =E_{\hat{\Lambda} \backslash \Lambda}\left(a_{\bar{\Lambda}} a_{\hat{\Lambda} \backslash \bar{\Lambda}}\right) \\
& =E_{\hat{\Lambda} \backslash \Lambda}\left(a_{\hat{\Lambda} \backslash \bar{\Lambda}} a_{\bar{\Lambda}}\right) \\
& =a_{\hat{\Lambda} \backslash \bar{\Lambda}} E_{\hat{\Lambda} \backslash \Lambda}\left(a_{\bar{\Lambda}}\right) .
\end{aligned}
$$

Namely, $E_{\hat{\Lambda} \backslash \Lambda}\left(a_{\bar{\Lambda}}\right) \in \mathfrak{A}_{\hat{\Lambda} \backslash \bar{\Lambda}}^{\prime} \wedge \mathfrak{A}_{\hat{\Lambda} \backslash \Lambda}$, which is the assertion.

Now let $\Lambda, \Lambda_{1}, \Lambda_{2} \in F$ with $\Lambda \subset \subset \Lambda_{1} \subset \Lambda_{2}$, and consider $E_{\Lambda_{i} \backslash \Lambda}, i=1,2$. Then the restriction of $E_{\Lambda_{2} \backslash \Lambda}$ to $\mathfrak{A}_{\Lambda_{1}}$ also leaves $\varphi_{\Lambda_{1}}$ invariant. Hence its range is globally invariant under the modular group $\sigma_{t}^{\Lambda_{1}}$ by Takesaki Theorem. ${ }^{24}$ Moreover, since this restriction is the identity on $\mathfrak{A}_{\Lambda_{1} \backslash \bar{\Lambda}}$ (hence by Lemma 3.2 , it satisfies $E_{\Lambda_{2} \backslash \Lambda}\left\lceil\mathfrak{A}_{\Lambda_{1}}=\right.$ $\left.\mathcal{E}_{\Lambda_{2} \backslash \Lambda} \otimes \operatorname{id}_{\mathfrak{A}_{\Lambda_{1} \backslash \bar{\Lambda}}}\right)$, its range is contained in $\mathfrak{A}_{\Lambda_{1} \backslash \Lambda}$. But

$$
E_{\Lambda_{2} \backslash \Lambda}\left(\mathfrak{A}_{\Lambda_{1}}\right) \subset \mathcal{R}\left(E_{\Lambda_{1} \backslash \Lambda}\right)
$$

as the last algebra is, by Theorem 5.1 of Ref. 3, the largest $\varphi_{\Lambda_{1}}$-expected subalgebra of $\mathfrak{A}_{\Lambda_{1} \backslash \Lambda}$ (that is the largest subalgebra of $\mathfrak{A}_{\Lambda_{1} \backslash \Lambda}$ left globally invariant by the modular group $\left.\sigma_{t}^{\Lambda_{1}}\right)$. Since for $i=1,2$,

$$
\mathcal{R}\left(E_{\Lambda_{i} \backslash \Lambda}\right)=\mathcal{R}\left(\mathcal{E}_{\Lambda_{i} \backslash \Lambda}\right) \otimes \mathfrak{A}_{\Lambda_{i} \backslash \bar{\Lambda}},
$$

we obtain

$$
\mathcal{R}\left(\mathcal{E}_{\Lambda_{2} \backslash \Lambda}\right) \subset \mathcal{R}\left(\mathcal{E}_{\Lambda_{1} \backslash \Lambda}\right) .
$$

Namely, $\left\{\mathcal{E}_{\hat{\Lambda} \backslash \Lambda}: \hat{\Lambda} \in \mathcal{F}, \hat{\Lambda} \supset \Lambda\right\}$ is a decreasing net of conditional expectations defined on the full matrix algebra $\mathfrak{A}_{\bar{\Lambda}}$, which converges to a conditional expectation by a standard martingale convergence theorem, see Theorem 3 of Ref. 26. Denoting

$$
\mathcal{E}_{\Lambda^{\prime}}:=\lim _{\hat{\Lambda} \uparrow \mathbb{Z}^{d}} \mathcal{E}_{\hat{\Lambda} \backslash \Lambda}
$$

and

$$
\mathfrak{B}_{\partial \Lambda}:=\mathcal{R}\left(\mathcal{E}_{\Lambda^{\prime}}\right) \subset \mathfrak{A}_{\partial \Lambda},
$$

we obtain a conditional expectation

$$
E_{\Lambda^{\prime}}:=\mathcal{E}_{\Lambda^{\prime}} \otimes \mathrm{id}_{\mathfrak{A}_{\bar{\Lambda}^{\prime}}}
$$

with

$$
E_{\Lambda^{\prime}}: \mathfrak{A} \mapsto I_{\mathfrak{A}_{\Lambda}} \otimes \mathfrak{B}_{\partial \Lambda} \otimes \mathfrak{A}_{\bar{\Lambda}^{\prime}}
$$

By construction, the net $\left\{E_{\Lambda^{\prime}}\right\}_{\Lambda \in \mathcal{F}}$ of conditional expectations leaves the state $\varphi$ invariant.

We have proved the most part of the following:

Theorem 3.3. Let $\varphi \in \mathcal{S}(\mathfrak{A})$ be a Markov state. 
For each $\Lambda \in F$ there exists a Umegaki conditional expectation $E_{\Lambda^{\prime}}$ on $\mathfrak{A}$, which projects into the complement of $\Lambda$.

Moreover, the net $\left\{E_{\Lambda^{\prime}}\right\}_{\Lambda \in \mathcal{F}}$ satisfies:

(i) $E_{\Lambda^{\prime}}\left(\mathfrak{A}_{\bar{\Lambda}}\right) \subset \mathfrak{A}_{\partial \Lambda}$,

(ii) $E_{\Lambda^{\prime}}\left\lceil\mathfrak{A}_{\bar{\Lambda}^{\prime}}=\mathrm{id}_{\mathfrak{A}_{\bar{\Lambda}^{\prime}}}\right.$,

(iii) $\varphi \circ E_{\Lambda^{\prime}}=\varphi$,

(iv) if $\Lambda_{1} \subset \Lambda_{2}$ then $E_{\Lambda_{2}^{\prime}} E_{\Lambda_{1}^{\prime}}=E_{\Lambda_{2}^{\prime}}$.

Proof. We have to show only the projectivity condition (iv). Let $\Lambda_{1} \subset \Lambda_{2} \subset \subset \hat{\Lambda}$, and consider the region $\Gamma:=\bar{\Lambda}_{1} \cup \bar{\Lambda}_{2}$. In order to recover the $E_{\Lambda_{i}^{\prime}}$, it is enough to consider the conditional expectations

$$
\mathcal{F}_{\hat{\Lambda} \backslash \Gamma}^{i}:=E_{\hat{\Lambda} \backslash \Lambda_{i}}\left\lceil\mathfrak{A}_{\Gamma} .\right.
$$

By maximality (Theorem 5.1 of Ref. 3), we get for each $\hat{\Lambda} \supset \Gamma$,

$$
\mathcal{R}\left(\mathcal{F}_{\hat{\Lambda} \backslash \Gamma}^{2}\right) \subset \mathcal{R}\left(\mathcal{F}_{\hat{\Lambda} \backslash \Gamma}^{1}\right)
$$

Namely, we have two decreasing nets $\left\{\mathcal{F}_{\hat{\Lambda} \backslash \Gamma}^{i}: \hat{\Lambda} \in \mathcal{F}, \hat{\Lambda} \supset \Gamma\right\}_{i=1,2}$ of conditional expectations whose fixed points satisfy (3.2). Hence, the martingale-limits

$$
\mathcal{F}_{\Gamma^{\prime}}^{i}:=\lim _{\hat{\Lambda} \uparrow \mathbb{Z}^{d}} \mathcal{F}_{\hat{\Lambda} \backslash \Gamma}^{i}, \quad i=1,2
$$

satisfy the analogous relation (3.2). The assertion follows as for $i=1,2$,

$$
E_{\Lambda_{i}^{\prime}}:=\mathcal{F}_{\Gamma^{\prime}}^{i} \otimes \operatorname{id}_{\mathfrak{A}_{\Gamma^{\prime}}} .
$$

\section{An Equivalent Formulation of the Quantum Markov Property in Terms of Umegaki Conditional Expectations}

It is of interest to investigate the converse of Theorem 3.3. This can be done by studying some finite-dimensional distributions associated to a state $\varphi$ consistent with a net $\left\{E_{\Lambda^{\prime}}\right\}$ of conditional expectations satisfying properties (i)-(iii) of Theorem 3.3.

Suppose we have a locally faithful state on the quasi-local algebra $\mathfrak{A}$, then a potential $h_{\Lambda}$ is canonically defined for each finite subset $\Lambda \in F$ as

$$
\varphi_{\Lambda}=\operatorname{Tr}_{\mathfrak{A}_{\Lambda}}\left(e^{-h_{\Lambda}} \cdot\right) .
$$

Such a set of potentials $\left\{h_{\Lambda}\right\}_{\Lambda \in F}$ satisfies normalization conditions

$$
\operatorname{Tr}_{\mathfrak{A}_{\Lambda}}\left(e^{-h_{\Lambda}}\right)=1
$$

together with compatibility conditions

$$
\left(\operatorname{Tr}_{\mathfrak{A}_{\hat{\Lambda} \backslash \Lambda}} \otimes \operatorname{id}_{\mathfrak{A}_{\Lambda}}\right)\left(e^{-h_{\hat{\Lambda}}}\right)=e^{-h_{\Lambda}}
$$

for $\Lambda \subset \hat{\Lambda}$. 
Let the locally faithful state $\varphi$ be consistent with a net $\left\{E_{\Lambda^{\prime}}\right\}$ of conditional expectations as that in Theorem 3.3. Let $\Lambda_{1} \subset \subset \Lambda_{2} \subset \subset \Lambda_{3}$ be fixed bounded regions of $\mathbb{Z}^{d}$.

Lemma 4.1. Under the above conditions we have

$$
\begin{aligned}
h_{\Lambda_{2}} & =H_{1}+\hat{H}_{1}, \\
h_{\Lambda_{2} \backslash \Lambda_{1}} & =H_{1}+\hat{H}, \\
h_{\Lambda_{3}} & =H_{2}+H_{2,1}+\hat{H}_{1},
\end{aligned}
$$

for self-adjoint operators $H_{i} \in \mathfrak{A}_{\Lambda_{i+1} \backslash \Lambda_{i}}, i=1,2 ; H_{2,1} \in \mathfrak{A}_{\bar{\Lambda}_{2} \backslash \Lambda_{1}} ; \hat{H}_{1} \in$ $\mathfrak{A}_{\bar{\Lambda}_{1}} ; \hat{H} \in \mathfrak{A}_{\partial \Lambda_{1}}$. Moreover, the above operators satisfy the following commutation relations:

$$
\begin{array}{cl}
{\left[H_{2}, H_{2,1}\right]=0,} & {\left[H_{2,1}, \hat{H}_{1}\right]=0} \\
{\left[H_{1}, \hat{H}\right]=0 ;} & {\left[H_{1}, \hat{H}_{1}\right]=0}
\end{array}
$$

Proof. The proof is quite similar to that of the implication (i) $\Rightarrow$ (ii) of Theorem 5.1 in Ref. 4. We verify the result for $h_{\Lambda_{3}}$, the others follow analogously. In such a situation,

$$
E_{\Lambda^{\prime}}=\mathcal{E}_{\Lambda^{\prime}} \otimes \operatorname{id}_{\mathfrak{A}_{\bar{\Lambda}^{\prime}}}
$$

for a transition expectation $\mathcal{E}_{\Lambda^{\prime}}: \mathfrak{A}_{\bar{\Lambda}} \mapsto \mathfrak{B}_{\partial \Lambda} \subset \mathfrak{A}_{\partial \Lambda}$.

Consider the (finite) sets $\left\{P_{\omega_{\partial \Lambda_{i}}}^{\partial \Lambda_{i}}\right\}, i=1,2$ consisting of the minimal central projections of the ranges $\mathfrak{B}_{\partial \Lambda_{i}}$ of the transition expectations $\mathcal{E}_{\Lambda_{i}^{\prime}}$. The state $\varphi$, when restricted to $\mathfrak{A}_{\Lambda_{3}}$, is determined by its value on the elements of the form

$$
\begin{aligned}
a= & \sum_{\omega_{\partial \Lambda_{2}}, \omega_{\partial \Lambda_{1}}} \iota_{\Lambda_{3}}\left(A \otimes P_{\omega_{\partial \Lambda_{2}}}^{\partial \Lambda_{2}}\left(a_{\omega_{\partial \Lambda_{2}}} \otimes \bar{a}_{\omega_{\partial \Lambda_{2}}}\right) P_{\omega \partial \Lambda_{2}}^{\partial \Lambda_{2}} \otimes B \otimes P_{\omega_{\partial \Lambda_{1}}}^{\partial \Lambda_{1}}\right. \\
& \left.\times\left(b_{\omega_{\partial \Lambda_{1}}} \otimes \bar{b}_{\omega_{\partial \Lambda_{1}}}\right) P_{\omega_{\partial \Lambda_{1}}}^{\partial \Lambda_{1}} \otimes C\right),
\end{aligned}
$$

where

$$
\begin{aligned}
& A \in \mathfrak{A}_{\Lambda_{3} \backslash \bar{\Lambda}_{2}}, \quad P_{\omega_{\partial \Lambda_{2}}^{\partial \Lambda_{2}}\left(a_{\omega_{\partial \Lambda_{2}}} \otimes \bar{a}_{\omega_{\partial \Lambda_{2}}}\right) P_{\omega_{\partial \Lambda_{2}}}^{\partial \Lambda_{2}} \in \mathfrak{A}_{\partial \Lambda_{2}},}, \\
& B \in \mathfrak{A}_{\Lambda_{2} \backslash \bar{\Lambda}_{1}}, \quad P_{\omega_{\partial \Lambda_{1}}^{\partial \Lambda_{1}}\left(b_{\omega_{\partial \Lambda_{1}}} \otimes \bar{b}_{\omega_{\partial \Lambda_{1}}}\right) P_{\omega_{\partial \Lambda_{1}}}^{\partial \Lambda_{1}} \in \mathfrak{A}_{\partial \Lambda_{1}},},
\end{aligned}
$$

and finally $C \in \mathfrak{A}_{\Lambda_{1}}$. In such a way,

$$
\begin{aligned}
\varphi(a)= & \left.\sum_{\omega_{\partial \Lambda_{2}}, \omega_{\partial \Lambda_{1}}} \varphi\left(\iota_{\Lambda_{3}}\left(A \otimes P_{\omega_{\partial \Lambda_{2}}^{\partial \Lambda_{2}}}^{\partial \Lambda_{\omega_{\partial \Lambda_{2}}}} \otimes I\right) P_{\omega_{\partial \Lambda_{2}}^{\partial \Lambda_{2}}}^{\partial \Lambda_{2}}\right)\right) \\
& \times \phi_{\omega_{\partial \Lambda_{2}}}^{\Lambda_{2}}\left(\bar{a}_{\omega_{\partial \Lambda_{2}}} \otimes B \otimes P_{\omega_{\partial \Lambda_{1}}^{\partial \Lambda_{1}}}^{\partial}\left(b_{\omega_{\partial \Lambda_{1}}} \otimes I\right) P_{\omega_{\partial \Lambda_{1}}^{\partial \Lambda_{1}}}^{\Lambda_{\partial}} \otimes I\right) \phi_{\omega_{\partial \Lambda_{1}}}^{\Lambda_{1}}\left(\bar{b}_{\omega_{\partial \Lambda_{1}}} \otimes C\right)
\end{aligned}
$$

with $\phi_{\omega_{\partial \Lambda_{i}}}^{\Lambda_{i}}, i=1,2$, states recovered according to $(2.2)$. 
Let the potentials $h_{\omega_{\partial \Lambda_{2}}}^{2}, h_{\omega_{\partial \Lambda_{2}}, \omega_{\partial \Lambda_{1}}}^{2}$ and $\hat{h}_{\omega_{\partial \Lambda_{1}}}^{1}$ be related to the states

$$
\begin{aligned}
A \otimes a_{\omega_{\partial \Lambda_{2}}} & \mapsto \varphi\left(\iota _ { \Lambda _ { 3 } } \left(A \otimes P_{\left.\omega_{\partial \Lambda_{2}}^{\partial \Lambda_{2}}\left(a_{\omega_{\partial \Lambda_{2}}} \otimes I\right) P_{\omega_{\partial \Lambda_{2}}}^{\partial \Lambda_{2}}\right),} \bar{a}_{\omega_{\partial \Lambda_{2}} \otimes B \otimes b_{\omega_{\partial \Lambda_{1}}}} \mapsto \phi_{\omega_{\partial \Lambda_{2}}}^{\Lambda_{2}}\left(\bar{a}_{\omega_{\partial \Lambda_{2}}} \otimes B \otimes P_{\omega_{\partial \Lambda_{1}}}^{\partial \Lambda_{1}}\left(b_{\omega_{\partial \Lambda_{1}}} \otimes I\right) P_{\omega_{\partial \Lambda_{1}}}^{\partial \Lambda_{1}} \otimes I\right),\right.\right. \\
\bar{b}_{\omega_{\partial \Lambda_{1}}} \otimes C & \mapsto \phi_{\omega_{\partial \Lambda_{1}}}^{\Lambda_{1}}\left(\bar{b}_{\omega_{\partial \Lambda_{1}}} \otimes C\right)
\end{aligned}
$$

respectively, according to a formula analogous to (4.1).

Now we define

$$
\begin{aligned}
H_{2} & :=\sum_{\omega_{\partial \Lambda_{2}}}\left(I \otimes P_{\omega_{\partial \Lambda_{2}}}^{\partial \Lambda_{2}}\right)\left(h_{\omega_{\partial \Lambda_{2}}}^{2} \otimes I\right)\left(I \otimes P_{\omega_{\partial \Lambda_{2}}}^{\partial \Lambda_{2}}\right), \\
H_{2,1} & :=\sum_{\omega_{\partial \Lambda_{2}}, \omega_{\partial \Lambda_{1}}}\left(P_{\omega_{\partial \Lambda_{2}}}^{\partial \Lambda_{2}} \otimes I \otimes P_{\omega_{\partial \Lambda_{1}}}^{\partial \Lambda_{1}}\right)\left(I \otimes h_{\omega_{\partial \Lambda_{2}}, \omega_{\partial \Lambda_{1}}}^{2} \otimes I\right)\left(P_{\omega_{\partial \Lambda_{2}}}^{\partial \Lambda_{2}} \otimes I \otimes P_{\omega_{\partial \Lambda_{1}}}^{\partial \Lambda_{1}}\right), \\
\hat{H}_{1} & :=\sum_{\omega_{\partial \Lambda_{1}}}\left(P_{\omega_{\partial \Lambda_{1}}^{\partial \Lambda_{1}}}^{\partial \Lambda_{1}} \otimes I\right)\left(I \otimes \hat{h}_{\omega_{\partial \Lambda_{1}}}^{1}\right)\left(P_{\omega_{\partial \Lambda_{1}}^{\partial \Lambda_{1}}}^{\partial \Lambda_{1}} \otimes I\right) .
\end{aligned}
$$

It is straightforward to verify that $H_{2}, H_{2,1}$ and finally $\hat{H}_{1}$ satisfy commutation relations (4.3), and give rise to $h_{\Lambda_{3}}$ through (4.2).

Now we are in a position to establish the converse of Theorem 3.3.

Theorem 4.1. Let a locally faithful state $\varphi \in \mathcal{S}(\mathfrak{A})$ be given, together with a net $\left\{E_{\Lambda^{\prime}}\right\}_{\Lambda \in \mathcal{F}}$ of Umegaki conditional expectations satisfying conditions (i)-(iii) of Theorem 3.3.

Then $\varphi$ is a Markov state in the sense of Definition 3.1.

Proof. The proof is quite similar to part (ii) $\Rightarrow$ (iii) of Theorem 5.1 of Ref. 4, taking into account Lemma 4.1. Namely, let $\Lambda_{1} \subset \subset \Lambda_{2}$ be fixed. Then the generalized conditional expectation

$$
E_{\Lambda_{2} \backslash \Lambda_{1}}^{\varphi_{\Lambda_{2}}}: \mathfrak{A}_{\Lambda_{2}} \mapsto \mathfrak{A}_{\Lambda_{2} \backslash \Lambda_{1}}
$$

leaving fixed $\varphi_{\Lambda_{2}}$ can be obtained as

$$
E_{\Lambda_{2} \backslash \Lambda_{1}}^{\varphi_{\Lambda_{2}}}(A)=\left(\operatorname{id}_{\mathfrak{A}_{\Lambda_{2} \backslash \Lambda_{1}}} \otimes \operatorname{Tr}_{\mathfrak{A}_{\Lambda_{1}}}\right)\left(k_{\Lambda_{2}, \Lambda_{1}}^{*} A k_{\Lambda_{2}, \Lambda_{1}}\right)
$$

where $k_{\Lambda_{2}, \Lambda_{1}}$ is the transition operator given by

$$
k_{\Lambda_{2}, \Lambda_{1}}=e^{-\frac{1}{2} h_{\Lambda_{2}}} e^{\frac{1}{2} h_{\Lambda_{2} \backslash \Lambda_{1}}} .
$$

Thus, by Lemma 4.1, we have

$$
k_{\Lambda_{2}, \Lambda_{1}}=e^{-\frac{1}{2} \hat{H}_{1}} e^{\frac{1}{2} \hat{H}} \in \mathfrak{A}_{\bar{\Lambda}_{1}},
$$

i.e. $E_{\Lambda_{2} \backslash \Lambda_{1}}^{\varphi_{\Lambda_{2}}}$ acts as the identity on $\mathfrak{A}_{\Lambda_{2} \backslash \bar{\Lambda}_{1}}$. 


\section{Extremal Disintegration of Markov States and the Relation with Equilibrium States}

When one starts with a locally faithful Markov state, he can recover a projective net $\left\{E_{\Lambda^{\prime}}\right\}_{\Lambda \in \mathcal{F}}$ of Umegaki conditional expectations projecting into complements $\Lambda^{\prime}$ of bounded regions $\Lambda \in \mathcal{F}$, and leaving invariant the state $\varphi$ (Theorem 3.3). In such a way, one can apply the results contained in Ref. 2, obtaining the extremal disintegration for the state $\varphi$. In addition, a Markov state is also an equilibrium state w.r.t. a suitable automorphism group $\alpha_{t}$. We show that almost all states appearing in the extremal disintegration of the Markov state $\varphi$ are also Markov states and equilibrium states w.r.t. the same automorphism group $\alpha_{t}$.

We start by showing that a (locally faithful) Markov state is automatically a KMS state w.r.t. an automorphism group canonically associated to $\varphi .^{\mathrm{c}}$ In such a way, we obtain the generalization to the multi-dimensional situation, of Theorem 5.3 of Ref. 4.

Theorem 5.1. Let $\varphi \in \mathcal{S}(\mathfrak{A})$ be a Markov state, and $h_{\Lambda}$ be recovered by (4.1).

Then the pointwise-norm limit

$$
\alpha_{t}(a):=\lim _{\Lambda \uparrow \mathbb{Z}^{d}} e^{-i \mathrm{th}_{\Lambda}} a e^{i \mathrm{th}_{\Lambda}}
$$

exists and defines a strongly continuous one-parameter automorphism group of the quasi-local algebra $\mathfrak{A}$ which admits $\varphi$ as a KMS state. Furthermore, $\varphi$ has a normal faithful extension on all of $\pi_{\varphi}(\mathfrak{A})^{\prime \prime}$.

In particular, any locally faithful Markov state is faithful.

Proof. Starting from $\Lambda_{1} \subset \subset \Lambda_{2} \subset \subset \Lambda_{3}$, the cocycle $e^{i \operatorname{th}_{\Lambda_{3}}} e^{-i \operatorname{th}_{\Lambda_{2}}}$ commutes with each element $a \in \mathfrak{A}$ localized in $\mathfrak{A}_{\Lambda_{1}}$ by Lemma 4.1. Now let $a \in \mathfrak{A}$ be localized in the bounded region $\Lambda_{a}$. By the above considerations, one concludes that $e^{-i \mathrm{th}_{\Lambda}} a e^{i \mathrm{th}_{\Lambda}}$ coincides with $e^{-i \mathrm{th}_{\bar{\Lambda}_{a}} a e^{i \mathrm{th}_{\bar{\Lambda}_{a}}}}$ for each bounded region $\Lambda$ such that $\bar{\Lambda}_{a} \subset \subset \Lambda$. Thus $e^{-i \mathrm{th}_{\Lambda}} a e^{i \mathrm{th}_{\Lambda}}$ becomes asymptotically constant ( $t$ fixed) on the localized elements $a \in \mathfrak{A}$, i.e. it trivially converges pointwise in norm, on the localized elements of $\mathfrak{A}$. Furthermore, by a standard $3-\varepsilon$ trick, it converges on all of $\mathfrak{A}$. It is straigthforward to verify that $t \mapsto \alpha_{t}$ is actually a group of automorphisms of $\mathfrak{A}$, which is also pointwise-norm continuous in $t$, i.e. a strongly continuous group of automorphisms of $\mathfrak{A}$. By constuction, $\varphi$ is automatically a KMS state for $\alpha_{t}$ at inverse temperature $\beta=-1$.

The last assertions follow by Corollary 5.3.9 of Ref. 10, taking into account that $\mathfrak{A}$ is a simple $C^{*}$-algebra (Proposition 2.6.17 of Ref. 9).

The disintegration theory of states which are invariant w.r.t. a net of conditional expectations was developed in Ref. 2 (see also Ref. 22 and 25). We will show these results in the situation under consideration.

\footnotetext{
${ }^{\mathrm{c}}$ Indeed, this automorphism group is canonically associated to the net $\left\{E_{\Lambda^{\prime}}\right\}_{\Lambda \in \mathcal{F}}$, see below.
} 
As a Markov state $\varphi$ is automatically invariant w.r.t. a net $\left\{E_{\Lambda^{\prime}}\right\}_{\Lambda \in \mathcal{F}}$ of conditional expectations which acts locally (Theorem 3.3 ), we can extend the $E_{\Lambda^{\prime}}$ to normal faithful conditional expectations $\varepsilon_{\Lambda^{\prime}}^{\varphi}$ acting on all of $\pi_{\varphi}(\mathfrak{A})^{\prime \prime}$. Namely, the conditional expectations defined as

$$
\varepsilon_{\Lambda^{\prime}}^{\varphi}:=\mathcal{E}_{\Lambda^{\prime}} \otimes \mathrm{id}_{\pi_{\varphi}\left(\mathfrak{A}_{\Lambda^{\prime}}\right)^{\prime \prime}}
$$

do the job.

In such a way, we have a decreasing net $\left\{\varepsilon_{\Lambda^{\prime}}^{\varphi}\right\}_{\Lambda \in \mathcal{F}}$ of normal faithful conditional expectations which converges pointwise in the strong operator topology, to a conditional expectation $\varepsilon_{\infty}^{\varphi}$ by a standard convergence result. ${ }^{26}$ As the algebra at infinity

$$
\mathfrak{Z}_{\varphi}^{\perp}:=\bigwedge_{\Lambda \in \mathcal{F}} \pi_{\varphi}\left(\mathfrak{A}_{\Lambda^{\prime}}\right)^{\prime \prime}
$$

coincides in our situation with the center

$$
\mathfrak{Z}_{\varphi}:=\pi_{\varphi}(\mathfrak{A})^{\prime} \wedge \pi_{\varphi}(\mathfrak{A})^{\prime \prime}
$$

of the representation (Theorem 2.6.10 of Ref. 9), we then obtain a conditional expectation

$$
\varepsilon_{\infty}^{\varphi}: \pi_{\varphi}(\mathfrak{A})^{\prime \prime} \mapsto \mathfrak{Z}_{\varphi}
$$

of $\pi_{\varphi}(\mathfrak{A})^{\prime \prime}$ onto $\mathfrak{Z}_{\varphi}$ which is precisely the conditional expectation described by Lemma 3.1 of Ref. 2. Furthermore, if $e_{\Lambda^{\prime}}^{\varphi}$ is the cyclic projection relative to $\mathcal{R}\left(\varepsilon_{\Lambda^{\prime}}^{\varphi}\right)$, the decreasing net $\left\{e_{\Lambda^{\prime}}^{\varphi}\right\}_{\Lambda \in \mathcal{F}}$ converges in the strong operator topology, to the corresponding cyclic projection $e_{\infty}^{\varphi}$ relative to $\mathcal{R}\left(\varepsilon_{\infty}^{\varphi}\right) \equiv \mathfrak{Z}_{\varphi}$.

In such a situation we have

Theorem 5.2. Let $\varphi \in \mathcal{S}(\mathfrak{A})$ be a Markov state. Consider the net $\left\{E_{\Lambda^{\prime}}\right\}_{\Lambda \in \mathcal{F}}$ of conditional expectations given in Theorem 3.3, together with the convex set $\mathcal{S}_{E}$ of its invariant states.

Then $\mathcal{S}_{E}$ is a Choquet simplex. Thus $\varphi$ admits the extremal disintegration

$$
\varphi=\int_{\partial \mathcal{S}_{E}} \psi \mu(d \psi),
$$

where $\mu$ is a unique Borel probability measure supported on the $G_{\delta}$-set $\partial \mathcal{S}_{E}$ consisting of all extremal invariant states. Moreover, the set $\partial \mathcal{S}_{E}$ consists of factor states.

Proof. It follows directly by Sec. I of Ref. 2, together with Sec. I.4 of Ref. 7, taking into account that $\mathfrak{A}$ is a separable $C^{*}$-algebra.

As a consequence of the above result, we have that the extremal disintegration (5.2) of a Markov state $\varphi$ coincides with the disintegration given in Theorem 5.3.30 of Ref. 10, when $\varphi$ is considered as a KMS state. 
Proposition 5.3. Let $\varphi \in \mathcal{S}(\mathfrak{A})$ be a Markov state.

Then the measure $\mu$ given in (5.2) (considered in a trivial way, as a measure on all of $\mathcal{S}(\mathfrak{A}))$ is identical to the central measure corresponding to $\varphi$. Moreover, the states $\psi$ appearing in the extremal disintegration (5.2) of $\varphi$, are almost surely $\left\{\alpha_{t}\right\}$-KMS states, where $\alpha_{t}$ is the automorphisms group of $\mathfrak{A}$ defined in (5.1).

Proof. By Theorem 3.2 of Ref. 2, the measure $\mu$ in (5.2), when it is trivially extended to all of $\mathcal{S}(\mathfrak{A})$, corresponds to the $\left\{\pi_{\varphi}(\mathfrak{A}),\left\{e_{\Lambda^{\prime}}^{\varphi}\right\}\right\}^{\prime}$-measure, see Proposition 4.3.3 of Ref. 9. But, in our situation we have

$$
\mathfrak{Z}_{\varphi}^{\perp}=\left\{\pi_{\varphi}(\mathfrak{A}),\left\{e_{\Lambda^{\prime}}^{\varphi}\right\}\right\}^{\prime}=\mathfrak{Z}_{\varphi}
$$

as the inclusion $\mathfrak{Z}_{\varphi}^{\perp} \subset\left\{\pi_{\varphi}(\mathfrak{A}),\left\{e_{\Lambda^{\prime}}^{\varphi}\right\}\right\}^{\prime}$ is trivial, and $\left\{\pi_{\varphi}(\mathfrak{A}),\left\{e_{\Lambda^{\prime}}^{\varphi}\right\}\right\}^{\prime} \subset \mathfrak{Z}_{\varphi}$ follows by the calculations similar to those in Theorem 4.3.14 of Ref. 9. ${ }^{\mathrm{d}}$

Since $\mu$ is the central measure, it is equal to the maximal measure corresponding to the disintegration of $\varphi$ considered as a $\left\{\alpha_{t}\right\}$-KMS state, see Theorem 5.3.30 of Ref. 10.

Summarizing, we collect all the above results in a theorem describing the main properties of a Markov state.

Theorem 5.4. Let $\varphi \in \mathcal{S}(\mathfrak{A})$ be a Markov state.

(i) The state $\varphi$ is an $\left\{\alpha_{t}\right\}-K M S$ state, where $\alpha_{t}$ is the automorphism group given in (5.1);

(ii) the state $\varphi$ admits a disintegration

$$
\varphi=\int_{\mathcal{S}(\mathfrak{A})} \psi \mu(d \psi),
$$

where the measure $\mu$ is concentrated on the factor states of $\mathfrak{A} ;{ }^{\mathrm{e}}$

(iii) almost all $\psi$ appearing in (5.3) are $\left\{\alpha_{t}\right\}$-KMS states, and Markov states in the sense of Definition 3.1.

Proof. It is immediate by the above results, taking into account that a KMS state on $\mathfrak{A}$ is automatically faithful.

Consider a Markov state $\varphi \in \mathcal{S}(\mathfrak{A})$. Then almost all $\psi$ appearing in the disintegration (5.3), are equilibrium states w.r.t. the same automorphism group given in (5.1). Thus, almost all $\psi$ are faithful.

d The fact that $\left\{\pi_{\varphi}(\mathfrak{A}),\left\{e_{\Lambda^{\prime}}^{\varphi}\right\}\right\}^{\prime}=\mathfrak{Z}_{\varphi}^{\perp}$ can be directly checked by $\left\{E_{\Lambda^{\prime}}\right\}$-asymptotic abelianess. Indeed, if $a \in \mathfrak{Z}_{\varphi}$, and commute with all $e_{\Lambda^{\prime}}^{\varphi}$, then

$$
e_{\infty}^{\varphi} a e_{\infty}^{\varphi}=\lim e_{\Lambda^{\prime}}^{\varphi} a e_{\Lambda^{\prime}}^{\varphi}=\lim a e_{\Lambda^{\prime}}^{\varphi}=a e_{\infty}^{\varphi} .
$$

Hence, $\varepsilon_{\infty}^{\varphi}(a)=a$ as $e_{\infty}^{\varphi}$ is cyclic for $\pi_{\varphi}(\mathfrak{A})$, i.e. $a \in \mathfrak{Z}_{\varphi}^{\perp}$.

This computation, which seems not explicitly stated, applies to the more general situations considered in Ref. 2.

e The measure $\mu$ is precisely the measure in $(5.2)$, trivially extended to all of $\mathcal{S}(\mathfrak{A})$. 
We conclude the present section with a formula which could have certain interest for physical applications.

Let $\varphi$ be a Markov state on the spin algebra. Then we can write the restriction $\varphi_{\Lambda}$ in a nice way as follows. We obtain for $A \in \mathfrak{A}_{\Lambda}$,

$$
\varphi\left(\iota_{\Lambda}(A)\right)=\sum_{\omega_{\partial \Lambda}}\left(\int_{\partial \mathcal{S}_{E}} \psi\left(\iota_{\partial \Lambda}\left(P_{\omega_{\partial \Lambda}}^{\partial \Lambda}\right)\right) \mu(d \psi)\right) \phi_{\omega_{\partial \Lambda}}^{\Lambda}(I \otimes A),
$$

where the $\psi$ are the states appearing in the extremal disintegration (5.2) of the Markov state $\varphi$ under consideration, and the $\phi_{\omega_{\partial \Lambda}}^{\Lambda}$ are the states associated to the conditional expectation $\mathcal{E}_{\Lambda^{\prime}}$ by $(2.2)$.

Notice that, under the identification

$$
\mathfrak{Z}_{\varphi} \sim L^{\infty}\left(\partial \mathcal{S}_{E}, \mu\right)
$$

the states $\psi$ in $(5.4)$ can be recovered as

$$
\psi(a)=\varepsilon_{\infty}^{\varphi}\left(\pi_{\varphi}(a)\right)(\psi), \quad \alpha \in \mathfrak{A}
$$

\section{Invariant Markov States}

As the translations act in a natural way on the spin algebra, one can ask for Markov states which are invariant w.r.t. the $\mathbb{Z}^{d}$-action.

Let $\tau_{x}: \mathfrak{A} \mapsto \mathfrak{A}$ be the shift generated in a natural way on the spin algebra by the translation $x \in \mathbb{Z}^{d}$, and consider a Markov state $\varphi \in \mathcal{S}(\mathfrak{A})$ invariant w.r.t. the $\tau_{x}$. In such a situation, one easily verifies that the generalized conditional expectations associated to the restrictions of $\varphi$ satisfy

$$
E_{\hat{\Lambda} \backslash \Lambda}^{\varphi_{\hat{\Lambda}}}=\tau_{x}^{-1} \circ E_{\hat{\Lambda}+x \backslash \Lambda+x}^{\varphi_{\hat{\Lambda}+x}} \circ \tau_{x} .
$$

Formula (6.1) directly yields for the corresponding Umegaki conditional expectations,

$$
E_{\Lambda^{\prime}}=\tau_{x}^{-1} \circ E_{\Lambda^{\prime}+x} \circ \tau_{x}
$$

Consider the dual action

$$
\omega \in \mathcal{S}(\mathfrak{A}) \mapsto T_{x} \omega:=\omega \circ \tau_{x} \in \mathcal{S}(\mathfrak{A})
$$

of $\mathbb{Z}^{d}$ on the state space of $\mathfrak{A}$.

Proposition 6.1. In the above situation, $T_{x}$ maps the set $\mathcal{S}_{E}$ of all $\left\{E_{\Lambda^{\prime}}\right\}$-invariant states of $\mathfrak{A}$ into itself, leaving globally stable the set $\partial \mathcal{S}_{E}$ of its extremal states.

Proof. If $\psi$ is $\left\{E_{\Lambda^{\prime}}\right\}$-invariant, then by $(6.2)$,

$$
\psi \circ \tau_{x} \circ E_{\Lambda^{\prime}}=\psi \circ E_{\Lambda^{\prime}+x} \circ \tau_{x}=\psi \circ \tau_{x}
$$

i.e. $\psi \circ \tau_{x}$ is $\left\{E_{\Lambda^{\prime}}\right\}$-invariant. Now let $\psi$ be $\left\{E_{\Lambda^{\prime}}\right\}$-extremal, and consider a convex decomposition

$$
\psi \circ \tau_{x}=\lambda \psi_{1}+(1-\lambda) \psi_{2}
$$


with $\psi_{1}, \psi_{2} \in \mathcal{S}_{E}$, and $0<\lambda<1$. With $b:=\tau_{x}(a)$ we get

$$
\psi(b)=\lambda \psi_{1}\left(\tau_{x}^{-1}(b)\right)+(1-\lambda) \psi_{2}\left(\tau_{x}^{-1}(b)\right) .
$$

Namely, we have just obtained a convex decomposition of $\psi$ by the $\left\{E_{\Lambda^{\prime}}\right\}$-invariant states $\psi_{i} \circ \tau_{x}^{-1}, i=1,2$. As $\psi$ is $\left\{E_{\Lambda^{\prime}}\right\}$-extremal, we conclude that $\psi_{1} \circ \tau_{x}^{-1}=\psi_{2} \circ \tau_{x}^{-1}=\psi$ or equivalently $\psi_{1}=\psi_{2}=\psi \circ \tau_{x}$, i.e. $T_{x} \psi$ is $\left\{E_{\Lambda^{\prime}}\right\}$-extremal as well.

Consider the $\left\{E_{\Lambda^{\prime}}\right\}$-extremal disintegration (5.3) of the $\left\{\tau_{x}\right\}$-invariant Markov state $\varphi$. We obtain

$$
\varphi=T_{x} \varphi=\int_{\mathcal{S}(\mathfrak{A})} T_{x} \psi \mu(d \psi)=\int_{\mathcal{S}(\mathfrak{A})} \psi \mu \circ T_{x}^{-1}(d \psi),
$$

that is by Proposition 6.1,

$$
\mu \circ T_{x}=\mu
$$

by the uniqueness of the maximal measure, see Theorem II.3.6. of Ref. 7. We then have a measure-preserving $\mathbb{Z}^{d}$-action $x \mapsto T_{x}$ on the state space $\mathcal{S}(\mathfrak{A})$ of $\mathfrak{A}$.

Fix our attention on an ergodic component $m$ of the ergodic disintegration

$$
\mu=\int m \nu(d m)
$$

of the measure $\mu$ (see e.g. p. 153 of Ref. 27). ${ }^{\text {f }}$ If the standard measure space $(\mathcal{S}(\mathfrak{A}), m)$ is essentially transitive for the action of $\mathbb{Z}^{d}$ (i.e. when $m$ is concentrated on a single orbit), then the $\mathbb{Z}^{d}$-space $(\mathcal{S}(\mathfrak{A}), m)$ is similar to the $\mathbb{Z}^{d}$-space $\left(\mathbb{Z}^{d} / H, \lambda\right)$ based on the homogeneous space $\mathbb{Z}^{d} / H$, see Theorem 4.12 of Ref. 20 (see Ref. 18 for the original result). Here $H \subset \mathbb{Z}^{d}$ is a (closed) subgroup of $\mathbb{Z}^{d}$, and $\lambda$ is a probability measure equivalent to the Haar measure on the Abelian group $\mathbb{Z}^{d} / H$.

In the $\left\{E_{\Lambda^{\prime}}\right\}$-extremal disintegration of a $\left\{\tau_{x}\right\}$-invariant Markov state $\varphi$, states with a smaller symmetry might occur. In our example, the surviving symmetry group is precisely the subgroup $H \subset \mathbb{Z}^{d}$. This is an instance of the phenomenon of symmetry breaking, see e.g. Refs. 10, 19 and 21 for quite similar situations and for technical details.

Finally, we would like to remark that most of the last analysis can be repeated mutatis mutandis when the symmetries of the model under consideration are described by a general locally compact second countable group.

\section{Acknowledgments}

F. F. would like to thank the Indian Statistical Institute of Bangalore, and in particular R. Bhat, for financial support and kind hospitality (September 2001) when the paper was completed.

${ }^{\mathrm{f}}$ The ergodic disintegration of the measure $\mu$ corresponds to the direct-integral disintegration (Theorem 8.21 of Ref. 25) of the von Neumann algebra $L^{\infty}(\mathcal{S}(\mathfrak{A}), \mu)$ w.r.t. the fixed-point subalgebra relative to the natural $\mathbb{Z}^{d}$-action on $L^{\infty}(\mathcal{S}(\mathfrak{A}), \mu)$. 


\section{References}

1. L. Accardi, Noncommutative Markov chains, in Proc. of Int. School of Math. Phys. Camerino (1974) 268-295.

2. L. Accardi, Local perturbations of conditional expectations, J. Math. Anal. Appl. 72 (1979) 34-68.

3. L. Accardi and C. Cecchini, Conditional expectations in von Neumann algebras and a theorem of Takesaki, J. Funct. Anal. 45 (1982) 245-273.

4. L. Accardi and F. Fidaleo, Nonhomogeneous Markov states and quantum Markov fields, J. Funct. Anal., in press.

5. L. Accardi and A. Frigerio, Markovian cocycles, Proc. R. Ir. Acad. 83 (1983) 251-263.

6. L. Accardi and V. Liebscher, Markovian KMS states for one dimensional spin chains, Infin. Dim. Anal. Quantum Probab. Related Tops. 2 (1999) 645-661.

7. E. M. Alfsen, Compact Convex Sets and Boundary Integral (Springer, 1971).

8. O. Bratteli, Inductive limits of finite dimensional $C^{*}$-Algebras, Trans. Amer. Math. Soc. 171 (1972) 195-234.

9. O. Bratteli and D. W. Robinson, Operator Algebras and Quantum Statistical Mechanics I (Springer, 1981).

10. O. Bratteli and D. W. Robinson, Operator Algebras and Quantum Statistical Mechanics II (Springer, 1981).

11. R. L. Dobrushin, The description of a random field by means of conditional probabilities and conditions of its regularity, Theor. Probab. Appl. 13 (1968) 197-224.

12. R. L. Dobrushin, Gibbsian random fields for lattice systems with pairwise interactions, Funct. Anal. Appl. 2 (1968) 292-301.

13. R. L. Dobrushin, The problem of uniqueness of a Gibbsian random field and the problem of phase transitions, Funct. Anal. Appl. 2 (1968) 302-312.

14. F. Fidaleo and T. Isola, Minimal conditional expectations for inclusions with atomic centres, Internat. J. Math. 7 (1996) 307-327.

15. J.-F. Havet, Espérance conditionelle minimale, J. Operator Theory 24 (1990) 33-55.

16. F. Hiai and M. Tsukada, Martingale convergence of generalized conditional expectations on von Neumann algebras, Trans. Amer. Math. Soc. 282 (1984) 791-798.

17. B. Kümmerer, A non-commutative ergodic theorem, Invent. Math. 46 (1978) 139-145.

18. G. W. Mackey, Ergodic theory and virtual groups, Math. Ann. 166 (1966) 187-207.

19. D. Petz, Quasi-uniform ergodic theorems in von Neumann algebras, Bull. London Math. Soc. 16 (1984) 151-156.

20. A. Ramsay, Virtual groups and group actions, Adv. Math. 6 (1971) 253-322.

21. D. Ruelle, Statistical Mechanics (Benjamin, 1969).

22. S. Sakai, $C^{*}$-Algebras and $W^{*}$-Algebras (Springer, 1971).

23. S. Stratila, Modular Theory in Operator Algebras (Abacus Press, 1981).

24. M. Takesaki, Conditional expectations in von Neumann algebras, J. Funct. Anal. 9 (1972) 306-321.

25. M. Takesaki, Theory of Operator Algebras I (Springer, 1979).

26. M. Tsukada, Strong convergence of martingales in von Neumann algebras, Proc. Amer. Math. Soc. 88 (1983) 537-540.

27. P. Walters, An Introduction to Ergodic Theory (Springer, 1982).

28. N. E. Wegge-Olsen, K-Theory and $C^{*}$-Algebras (Oxford Univ. Press, 1993). 